intestinal absorption, enterohepatic cycling, distribution and hepatic conjugation. This would explain the rapid and of intense decline in levels, in spite of the high dose of antiepileptic, and the difficulty reversing the situation.

Conclusions Given the magnitude of the reduction in plasma levels, the speed with which it appears and the difficulty of getting it back at TI, we think that monitoring and dose adjustments are not useful to manage this interaction. A change of anticonvulsivant or antibiotic treatment should be considered.

No conflict of interest.

\section{PHC-011 DUAL ABSORPTION IN INTRANASAL ADMINISTRATION: A NEW PHARMACOKINETIC MODEL}

\section{doi:10.1136/ejhpharm-2013-000276.356}

${ }^{1} \mathrm{C}$ Neef, ${ }^{1} \mathrm{~N}$ Veldhorst-Janssen, ${ }^{2} \mathrm{~N}$ Punt, ${ }^{3} \mathrm{PHM}$ Van der Kuy, ${ }^{4} \mathrm{M}$ Marcus. 'Maastricht University Medical Center, Clinical Pharmacy \& Toxicology, Maastricht, The Netherlands; ${ }^{2}$ Maastricht University, Toxicology, Maastricht, The Netherlands; ${ }^{3}$ Orbis Medical Center, Pharmacy, Sittard-Geleen, The Netherlands; ${ }^{4}$ Maastricht University Medical Center, Anaesthesiology, Maastricht, The Netherlands

Background The role of pharmacokinetic modelling is important in the development of new formulations. Some of these models are related to a particular dosage form, others are similar to models that have already been developed. Intranasal (IN) administration can be an example of a dosage form with a specific pharmacokinetic model, especially when it is applied to create a systemic effect.

Purpose To design a pharmacokinetic model that adequately describes a dual absorption profile of the concentration-time curve for intranasal administration.

Materials and Methods A strategy to predict dual absorption was developed to describe the pharmacokinetics of an intranasal administration (model1 and model2). A programme for fitting and simulation was developed (SIMLAB). Midazolam nasal spray was used as an example for this model. To validate the final pharmacokinetic model, Monte Carlo simulations were performed.

Results We had trouble fitting the observations to a single onecompartment dual absorption model. In many cases a flip-flop condition occurred in which the fitted absorption rate was lower than the estimated elimination rate, and the elimination rate showed an unrealistic value. To prevent this flip-flop condition, we used the absorption parameters from the associated observations. We developed the following model: the model superposes two onecompartment absorption models where the dose is split up over the two compartment inputs and the concentration-time curves are separated by using different lag-times ( $\mathrm{t} 0$ ). Monte Carlo simulations resulted in a plasma concentration-time profile, indicating the median concentration and the 5th-95th percentile ranges. Biphasic profiles were observed starting at a parameter error of $15 \%$, increasing to $13.6 \%$ of biphasic profiles at a parameter error of $50 \%$. When increasing the difference between a parameter in Model 1 and Model 2, the contribution of t0 to creating a local minimum exceeded the contribution of $\mathrm{ka}$. The AUC of the measured and estimated curve was $201.6 \mu \mathrm{g} / \mathrm{L}^{*} \mathrm{~h}$ and $201.3 \mu \mathrm{g} / \mathrm{L}^{*} \mathrm{~h}$, respectively.

Conclusions The model developed is able to fit concentrationtime curves showing individual dual absorption curves adequately.

No conflict of interest.

\section{PHC-012 ERLOTINIB IN NON-SMALL CELL LUNG CANCER PATIENTS FROM FERNANDO FONSECA HOSPITAL}

doi:10.1136/ejhpharm-2013-000276.357

J Fernandes, P Frade, S Teixeira, R Afonso. Hospital Fernando Fonseca, Pharmacy, Amadora, Portugal
Background The oral epidermal growth factor receptor (EGFR) tyrosine kinase inhibitor (TKI) erlotinib is an established secondline treatment for advanced non-small cell lung cancer (NSCLC). Erlotinib delays disease progression and increases survival after first-line chemotherapy in patients with advanced NSCLC as second-line treatment. Maintenance treatment with erlotinib, when compared to placebo, could be associated with a significantly longer progression-free survival and tolerability mainly in EGFRactivating mutation tumours. However second-line treatment with erlotinib is not more effective than chemotherapy (pemetrexed or other). In terms of traditional toxicities associated with chemotherapy, erlotinib seems to have a better safety profile than chemotherapy, with no haematological toxicities. The most common event has been mild to moderate skin rash which is relatively manageable.

Purpose To study erlotinib's efficacy profile in Fernando Fonseca hospital NSCLC patients.

Materials and Methods We followed up 30 NSCLC patients, who had taken erlotinib before and after other approved chemotherapies, during the 14 months starting from June 2011. During this period we collected patient demographics and baseline characteristics and also their EGFR mutational status. To determine erlotinib effectiveness we calculated progression-free survival (PFS) which was defined as the time from starting erlotinib treatment to the date of documented disease progression or death.

Results The median age of our 30 patients was 62.5 years. The most common pathological subtype was adenocarcinoma $(66.7 \%)$. $46.6 \%$ of our patients had received one prior chemotherapy regimen before erlotinib and $36.6 \%$ had received two prior chemotherapy regimens before erlotinib. Two patients took erlotinib as a first line treatment. Median PFS for second-line erlotinib patients was 18.7 weeks while for third-line erlotinib patients it was 12.3 weeks. Only $50 \%$ of our patients had information available regarding EGFR mutational status; however patients who harboured tumour-associated EGFR activating mutations seemed to have higher response rates to erlotinib. Rash was the most common treatment-related adverse event with erlotinib, as expected.

Conclusions Our results show that maybe there could be a better disease outcome for advanced NSCLC patients if the oral epidermal growth factor receptor tyrosine kinase inhibitor (erlotinib) were administered as a second-line treatment instead of using it as a third-line treatment. As far as EGFR mutational status is concerned it seems that enhanced efficacy is related to EGFR mutation-positive disease.

No conflict of interest.

\section{PHC-013 EXPERIENCE WITH CANNABINOID TREATMENT}

doi:10.1136/ejhpharm-2013-000276.358

MT Martín, M Gómez, MS Rivero, MR Garrido, LC Fernández. Hospital San Pedro Alcántara, Pharmacy Service, Caceres, Spain

Background Since March 2011 cannabinoids have been authorised in Spain for the treatment of spasticity due to multiple sclerosis (MS). The product is composed primarily of two cannabinoids: CBD (cannabidiol) and THC (delta 9 tetrahydrocannabinol) and it is administered as a metered dose oro-mucosal spray. The dose should be individualised after a titration period.

Purpose To describe the use of CBD-THC in our hospital and to evaluate adverse effects and the quality of life of the patients treated.

Materials and Methods Descriptive study of all patients treated with CBD-THC from March 2011 to September 2012.

Patients were monitored from the start of their treatment. We recorded the titration period, maintenance dose and adverse 
reactions for each patient, besides demographic data. They answered a of quality of life questionnaire (SF-36) at the beginning of treatment and two months before starting.

Results During this period, 7 patients began treatment with CBDTHC, prescribed by neurologists. The average age was 40 years $( \pm 8.2), 4$ males and 3 females.

It was used for spasticity due to MS in two patients and it was off-label use for the rest of patients: two cases of refractory spasticity not caused by MS and three cases of neuropathic pain.

The quality of life improved $21 \%$, showed by SF-36 questionnaire.

The average titration period was 26 days, the average dose used was 7.8 sprays/day (standard deviation 3.27 ) (min: $3 \max 12$ ), spread three times a day.

All patients, except for one, suffered adverse reactions, mainly mild or moderate dizziness (57\% of them), dysgeusia (taste alteration) $29 \%$ and hypotension (14\%).

Conclusions The quality of life has improved for our patients treated with CBD-THC

As many adverse effects appeared and it was difficult to manage this drug the pharmacist's role assumed considerable importance; monitoring and pharmaceutical care is very necessary

No conflict of interest.

\section{PHC-014 EXPLORATORY ANALYSIS OF 1,936 SNPS IN 225 ADME GENES FOR ASSOCIATION WITH BUSULFAN CLEARANCE IN ADULT HEMATOPOIETIC STEM CELL RECIPIENTS}

doi:10.1136/ejhpharm-2013-000276.359

MH ten Brink, JJ Swen, JAM Wessels, T van der Straaten, J Zwaveling, HJ Guchelaar. Leiden Univerisity Medical Center, Clinical Pharmacy and Toxicology, Leiden, The Netherlands

Background Busulfan is used in preparative regimens prior to stem cell transplantation (SCT). There is significant inter-patient variability in busulfan pharmacokinetics (PK) and outcome is related to exposure.

To date, only polymorphisms in genes encoding for glutathioneS-transferases have been studied; they could only explain a small portion of the variability in $\mathrm{PK}$.

Purpose To investigate the role of other genetic variants on busulfan clearance by interrogating 1,936 variants in 225 genes that are involved in drug absorption, distribution, metabolism and excretion (ADME).

Materials and Methods 62 adult patients who received busulfan were genotyped using the Drug Metabolizing Enzymes and Transporters (DMET) array. Busulfan clearance was estimated with a limited sampling $(t=2.5,4 \mathrm{hrs})$ PK model. Individual SNPs were associated with busulfan clearance. Top SNPs and haplotypes were replicated in an independent cohort $(n=78)$.

Results In the discovery cohort 7 variants (3 SNPs and 4 haplotypes) explained 64\% (adjusted R2) of variance in busulfan clearance $(p<0.001)$. These genetic variants, located in GSTA5, CYP2C19, CYP39A1 (2 haplotypes), ABCB4, SLC22A4 and SLC7A8, were replicated in the second cohort. One haplotype in GSTA5 (rs4715354 and rs7746993) remained statistically significant $(\mathrm{P}=0.025)$ for correlation with busulfan clearance.

Conclusions This is the first study using an exploratory pharmacogenetic approach in 225 genes involved in ADME to explain the inter-individual variability in busulfan clearance. The GSTA5 haplotype was significantly correlated with busulfan clearance, both in the discovery and replication cohort. No additional genetic markers involved in drug metabolism and transport appear to be associated with busulfan clearance.

No conflict of interest.

\section{PHC-015 IMPACT OF MDR1 POLYMORPHISMS ON THE ANALGESIC EFFICACY OF TRAMADOL IN PATIENTS AFTER MINOR SURGERY}

doi:10.1136/ejhpharm-2013-000276.360

${ }^{1} \mathrm{H}$. Bakhouche, ${ }^{2} \mathrm{O}$ Matouskova, ${ }^{3} \mathrm{O}$ Polanecky, ${ }^{3} \mathrm{~J}$ Adamkova, ${ }^{3} \mathrm{~S}$ Adamek ${ }^{3},{ }^{4} \mathrm{O}$ Slanar. ${ }^{1}$ General Teaching Hospital Charles University in Prague (Vseobecná fakultní nemocnice), Hospital pharmacy, Prague, Czech Republic; ${ }^{2}$ First Faculty of Medicine and General Teaching Hospital Charles University in Prague, Institute of Pharmacology, Prague, Czech Republic; ${ }^{3}$ First Faculty of Medicine and University Hospital Motol Charles University in Prague, Third Department of Surgery, Prague, Czech Republic; ${ }^{4}$ First Faculty of Medicine and General Teaching Hospital Charles University in Prague, Institute of Pharmacology, Prague, Czech Republic

Background P-glycoprotein is a transmembrane transporter coded by the ATP-binding cassette sub-family B multi-drug resistance gene (MDR-1) gene. It influences the bioavailability, disposition and excretion of many drugs. Among the 50 SNPs of the MDR1 gene, more attention has been focused on the SNP at position 3435 in exon 26. Homozygous TT samples were associated with more than two-fold lower intestinal MDR1 expression levels compared with homozygous CC samples. A trial in patients suffering from chronic and cancer pain reported decreased opioid consumption in carriers of the 3435T allele. Our previous data suggest that the pharmacokinetics and therefore effectiveness of tramadol could be affected by MDR1 polymorphism C3435T.

Purpose To evaluate the possible effect of MDR1 polymorphisms on the analgesic efficacy of tramadol in realistic clinical settings.

Materials and Methods Pain intensity was assessed using a visual analogue scale at 2 and 24 hours after minor surgery in 156 patients. Polymorphisms and gene duplication in the MDR1 gene were analysed by PCR-RFLP (restriction fragment length polymorphism).

Results Variant allele $3435 \mathrm{~T}$ was seen at a frequency of $58.3 \%$. There were no statistically significant differences between MDR1 subgroups in basic demographic parameters. Mean VAS2h in groups C3435CC, C3435CT and C3435TT were $40.0 \pm 11.8$; $43.2 \pm 17.9$, resp. $45.5 \pm 16.1 \mathrm{~mm}(\mathrm{P}=\mathrm{ns})$. Corresponding values for mean pain difference, defined as VAS2-24h were $19.3 \pm 12.1 ; 21.3 \pm 14.6$ and $23.4 \pm 15.4 \mathrm{~mm}(\mathrm{P}=\mathrm{ns})$. Mean tramadol consumption was $2.47 \pm 1.17$, resp. $2.62 \pm 1.1 ; 2.42 \pm 1.1 ; 2.44 \pm 1.3 \mathrm{mg} / \mathrm{kg}(\mathrm{P}=\mathrm{ns})$ during the $24 \mathrm{~h}$ period. There were no significant differences in the drug consumption, reporting of adverse reactions or need for rescue analgesics among the MDR1 genotype subgroups.

Conclusions Although there were approximately 20\% higher mean pain difference values in the 3435TT group in comparison with the wild-type subjects, the between-group variation did not reach statistical significance.

No conflict of interest.

\section{PHC-016 NILOTINIB VERSUS IMATINIB FOR THE TREATMENT OF PATIENTS WITH NEWLY-DIAGNOSED PHILADEPHIA CHROMOSOME-POSITIVE, CHRONIC MYELOID LEUKAEMIA IN THE CHRONIC PHASE}

doi:10.1136/ejhpharm-2013-000276.361

'A Romania, ${ }^{2} \mathrm{~A}$ Buemi. 'University of Catania, Facoltà di Farmacia, Catania, Italy; ${ }^{2}$ A.R.N.A.S. Garibaldi - Nesima, Pharmacy Department, Catania, Italy

Background Nilotinib is a BCR-ABL inhibitor designed to be more potent and selective than imatinib. Imatinib was the first of a new class of drugs that act by specifically inhibiting a tyrosine kinase receptor.

Purpose To assess the molecular response at 12 months from the start of nilotinib treatment, defined as BCR-ABL transcript levels on the International Scale of $0 \cdot 1 \%$ or less by real-time quantitative PCR in a peripheral blood sample. 